\title{
Development of the Italian Version of the Oswestry Disability Index (ODI-I)
}

\author{
A Cross-Cultural Adaptation, Reliability, and Validity Study
}

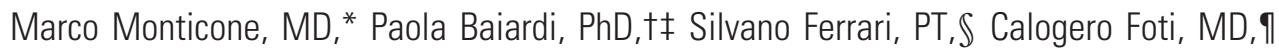 \\ Raffaele Mugnai, PT, || Paolo Pillastrini, PT, || Carla Vanti, PT, ${ }^{* *}$ and Gustavo Zanoli, MD††
}

Study Design. Evaluation of the psychometric properties of a translated, culturally adapted questionnaire.

Objective. Translating, culturally adapting, and validating the Italian version of the Oswestry Disability Index (ODI-I), allowing its use in Italian-speaking patients with low back pain inside and outside Italy.

Summary of Background Data. Growing attention is devoted to standardized outcome measures to improve interventions for low back pain. A translated form of the ODI in patients with low back pain has never been validated within the Italian population.

Methods. The ODI-I questionnaire was developed involving forward-backward translation, final review by an expert committee and test of the prefinal version to establish as better as possible proper correspondence with the original English latest version (2.1a). Psychometric testing included factor analysis, reliability by internal consistency (Cronbach $\alpha$ ) and test-retest repeatability (Intraclass Coefficient Correlation), concurrent validity by comparing the ODI-I to Visual Analogue Scale, (Pearson correlation), and construct validity by comparing the ODI-I to Roland Morris Disability Questionnaire, RMDQ, and to Short Form Health Survey, Short Form Health Survey-36 (Pearson correlation).

Results. The authors required a 3-month period before achieving a shared version of the ODI-I. The questionnaire was administered to 126 subjects, showing satisfying acceptability. Factor analysis demonstrated a 1-factor structure ( $45 \%$ of explained variance). The questionnaire showed high internal consistency ( $\alpha=0.855)$ and good test-retest reliability (ICC $=0.961)$. Concurrent validity was confirmed by a high correlation with Visual Analogue Scale $(r=0.73, P<0.001)$, Construct validity revealed high correlations with RMDQ $(r=0.819, P<0.001)$, and

From the *Physical Medicine and Rehabilitation Unit, Scientific Institute of Lissone, Institute of Care and Research, Salvatore Maugeri Foundation, IRCCS, Lissone, Milan, Italy; †Consorzio Valutazioni Biologiche e Farmacologiche, Pavia University, Pavia, Italy; $\ddagger$ Salvatore Maugeri Foundation, IRCCS, Pavia, Italy; $\mathbb{S}$ Manual Therapy Sciences, Padova University, Padova, Italy; IUUniversity of Rome Tor Vergata, Rome, Italy; |Department of Internal Medicine, S. Orsola-Malpighi Hospital, Bologna University, Bologna, Italy; * School of Physiotherapy, Bologna University, Bologna, Italy; ††Ferrara University and Casa di Cura SM Maddalena, Rome, Italy.

Acknowledgment date: December 11, 2008. Acceptance date: March 23, 2009.

The manuscript submitted does not contain information about medical device(s)/drug(s).

No funds were received in support of this work. No benefits in any form have been or will be received from a commercial party related directly or indirectly to the subject of this manuscript.

The Scientific and Technical Committee of the Salvatore Maugeri Foundation approved this research.

Address correspondence and reprint requests to Marco Monticone, MD, Via Monsignor Bernasconi, 16-20035 Lissone, Milan, Italy; E-mail:marco.monticone@fsm.it with Short Form Health Survey-36 domains, highly significant with the exception of Mental Health $(r=-0.139$, $P=0.126)$.

Conclusion. The ODI outcome measure was successfully translated into Italian, showing good factorial structure and psychometric properties, replicating the results of existing language versions of the questionnaire. Its use is recommended in research practice.

Key words: Oswestry Disability Index, Low Back Pain, Italian validation, outcome measures, psycho-metric properties. Spine 2009;34:2090-2095

Low back pain (LBP) constitutes a major health burden among western countries as well as a major cause of medical expenses, work absenteeism and disability. Lifetime prevalence of LBP is reported as over $70 \%$ in industrialized populations; peak prevalence occurs between ages 35 and $55 .^{1}$

Nearly $85 \%$ of the patients with a pain in their low back seen by primary care practitioners are affected by nonspecific LBP, defined as a pain not attributed to recognizable specific pathologies such as fractures, nerve root compressions, infections, tumors, inflammatory or systemic diseases. ${ }^{2,3}$ Nonspecific LBP is often influenced by heavy physical work, incorrect physical activities (e.g., frequent bending, twisting, lifting, pulling and pushing, and repetitive work), wrong postures, and psychosocial factors (e.g., distress, mood alterations, cognitive dysfunction, illness behavior, job dissatisfaction)., ${ }^{2,4}$

With such a high epidemiological and clinical burden, it is of great importance to apply evidence-based, validated and comprehensive outcome measures to help clinicians to quantify and improve interventions for LBP. ${ }^{5}$ As supported by most researchers, a number of diseasespecific measures are available for assessing functional outcomes related to $\mathrm{LBP}^{6}$ : among back related function outcome measures Oswestry Disability Index (ODI) and Roland-Morris Disability Questionnaire (RMDQ) were recommended. ${ }^{7}$

Initially developed by John O'Brien in 1976, ODI 1.0 version was first published in $1980^{8}$; the questionnaire was later adapted by the American Academy of Orthopedic Surgeons, omitting sections 1, 8, and 9 and changing the score of each item from 0 to 5 to 1 to $6 .^{9}$ The 2.0 version (1989) was a modification of the original scale made by a Medical Research Council group in the United Kingdom. ${ }^{10}$ Minor revisions were introduced in ODI version 2.1 (2000) for section 4 (walking) ${ }^{7}$; on September 2006, ODI 2.1a version was introduced, modifying instruc- 
tions of the first paragraph to consistently use present tense, as outlined at the website of the developers. ${ }^{11}$

The ODI represents a ten 6-point questionnaire. The first section rates the intensity of pain and the remaining ones cover the disabling effect of pain on typical daily activities: personal care, lifting, walking, sitting, standing, sleeping, sex life, social life, and traveling. Each item ranges form 0 to 5 and the sum of the 10 scores is expressed as a percentage of the maximum scores, varying from 0 (no disability) to 100 (maximum disability). The questionnaire is completed in about 5 minutes and scored in less than 1 minute. $^{7}$

The psychometric properties of the original version of the ODI were tested in a wide variety of clinical conditions demonstrating satisfying degree of internal consistency, reproducibility as well as face, content, concurrent, and construct validity. ${ }^{9}$

To authors' knowledge, the ODI was already validated in Greek, ${ }^{12}$ Norwegian, ${ }^{13}$ Japanese, ${ }^{14}$ Turkish, ${ }^{15}$ Korean, ${ }^{16}$ Arabic, ${ }^{17}$ German, ${ }^{18,19}$ Danish, ${ }^{20}$ Iranian, ${ }^{21}$ and Brazilian. ${ }^{22}$ These studies deserved great interest as they contributed to confirm reliability and validity of the translated forms of the questionnaire, allowing comparison of results, investigating pain, disability and functional status across different people and countries.

Though the Italian version of NASS/American Academy of Orthopedic Surgeons questionnaire was published, ${ }^{23}$ a validation trial of a translated form of the ODI was never conducted within an Italian population. As this lack represented a limit for clinicians and researchers of our country to share validated outcomes, the aim of this study was to describe translation, cultural adaptation, and validation (internal consistency, reproducibility, and validity) of the Italian version of the Oswestry Disability Index (ODI-I), in its latest version named 2.1a.

\section{- Materials and Methods}

The Institutional Review Board of the Institute approved the trial, allowing the development of the ODI-I.

\section{Subjects}

Outpatients referring to the Physical and Rehabilitation Medicine Units of a Research Hospital and a University Hospital of Italy were included into the study from May to September 2008.

Inclusion criteria were: common low back pain in its subacute (pain lasting more than 4 weeks) and chronic phase (pain lasting more than 12 weeks), adult age (18 years or older), ability to read and speak Italian fluently. Exclusion criteria were: acute common low back pain (included recent thoracolumbar trauma), specific causes of low back pain (disc herniation, lumbar stenosis, spinal deformity, fracture, spondylolisthesis), central or peripheral neurologic signs, systemic illness (tumor and rheumatologic diseases), psychiatric and mental deficits. Patients with recent cerebro-vascular accidents and myocardial infarctions were also excluded.

All the patients included were investigated for demographic and clinical characteristics. A specific schedule was prepared to collect main comorbidities. All the patients eligible gave their written consent to be involved into the study.

\section{Translation and Cross-Cultural Adaptation}

This stage followed the Guidelines for the Process of CrossCultural Adaptation of Self-Report Measures. ${ }^{24,25}$

Step 1. Forward Translation. ODI 2.1a was initially forward translated from English into Italian. The purpose was to retain the concept of the original scale, using culturally and clinically fitting expressions. Two translations were performed independently by translators with Italian as their native tongue; one of them (naive translator) was not familiar with the measure. Keeping the language colloquial and compatible with a reading age level of 14 years, poorer wording choices were outlined and resolved in a discussion between the 2 translators. With regard to section 4 (walking), walking distances described in terms of miles or yards (e.g., " 1 mile," " $1 / 2$ mile," "100 yards") were judged unfamiliar to the Italians: the distances were changed to kilometers or meters (respectively, 1 kilometer, $500 \mathrm{~m}$, and $100 \mathrm{~m})$. Step 1 ended when a common adaptation was shared. None of the items was excluded.

Step 2. Backward Translation. Two bilingual native Englishspeaking translators backward translated the initial translation; the translators were selected because unaware of concepts explored and without medical background. Taking into account cultural diversities, conceptual equivalence, or vocabulary differences, the aim was to make sure that the Italian version reflected the same item contents of the original version.

Step 3. Expert Committee. The translated versions were submitted to a bilingual committee composed of clinicians, methodologists, and psychometricians; the 4 translators were included. To identify difficulties, inconsistencies or mistakes in translation, the committee explored semantic, idiomatic, experiential and conceptual equivalence of items and answers options. Moreover, the committee took into consideration the Italian version of ODI 2.1, formerly translated by one of the authors of this work (G.Z.), ${ }^{11}$ but neither back warded nor validated. Step 3 ended when a prefinal version was achieved.

Step 4. Test of the Prefinal Version. The scale was delivered to 30 low back pain sufferers. This field test had the aim to probe what was meant by each item and the chosen response; the distribution of responses was also checked for missing items. All the findings from this step were re-evaluated by the expert committee, although no adjustment was further required.

Step 5. Submission to the Developer. The final shared version of the questionnaire (see Appendix, Supplemental Digital Content 1, http://links.lww.com/BRS/A381) was sent to the developer.

\section{Psychometric Scale Properties}

Acceptability. Time needed to answer the questionnaire was registered. Once completed, patients were asked about troubles encountered; at the same time, examiners checked all data, included missing or multiple responses.

Factor Analysis. The factor structure of the ODI-I was analyzed by means of a factor analysis. Cattel Scree Test was used to determine the number of extracted factors (eigenvalues greater than 1).

Reliability. This psychometric property was performed by means of internal consistency and test-retest stability. The first characteristic was examined with Cronbach $\alpha$ estimated for the whole questionnaire. The second characteristic was analyzed asking the patients to complete the Questionnaire 7 days later 
the first fulfillment; Intraclass Correlation Coefficient (ICC) was applied for testing agreement between baseline and 7 day ODI-I scores as well as to evaluate item by item agreement.

Validity. Concurrent validity was achieved comparing (Pearson correlations) ODI-I to Visual Analogue Scale (VAS) ${ }^{26}$; construct validity was achieved comparing (Pearson correlations) ODI-I to Roland Morris Disability Questionnaire (RMDQ) $)^{27,28}$ and to Short Form Health Survey (SF-36). ${ }^{29,30}$

\section{Statistical Methods}

All statistical analyses were performed using SPSS, 15.0 (Italian version, Appendix 1, Supplemental Digital Content 1, http://links.lww.com/BRS/A381).

\section{Results}

\section{Subjects}

The trial included a population of 126 subjects, 73 females $(58 \%)$ and 53 males (42\%), mean age $47 \pm 14$ years (range: $18-89$ ). Mean low back pain duration was $20.9 \pm 19.6$ months (range: $1-108$ ).

Additional socio-demographic characteristics of the enrolled population are described in Table 1.

\section{Translation and Cross-Cultural Adaptation}

A forward backward translation was used to translate the questionnaire into Italian, involving 4 translators. A 2 -month period was necessary before a culturally adapted version was reached. Further revision conducted by selected experts in spinal and psychometric research as well as a prefinal version testing (1-month length) confirmed the work done.

Table 1. Socio-Demographic Characteristics of the Population

\begin{tabular}{|c|c|c|}
\hline Variable & $\mathrm{N}$ & $\%$ \\
\hline Married & 87 & 69 \\
\hline Employed & 103 & 81.7 \\
\hline Smoking & 27 & 21.4 \\
\hline \multicolumn{3}{|l|}{ Frequency of pain } \\
\hline Sometimes & 33 & 26.2 \\
\hline Often & 68 & 54.0 \\
\hline Persistent & 25 & 19.8 \\
\hline Leg pain & 67 & 53.1 \\
\hline \multicolumn{3}{|l|}{ Drugs utilization } \\
\hline Antidepressants & 3 & 2.4 \\
\hline Analgesics & 50 & 39.7 \\
\hline Muscle relaxants & 2 & 1.6 \\
\hline NSAIDs & 28 & 22.2 \\
\hline None & 43 & 34.1 \\
\hline \multicolumn{3}{|l|}{ Education level } \\
\hline Elementary & 5 & 4.0 \\
\hline Mid School & 17 & 13.5 \\
\hline Upper school & 59 & 46.8 \\
\hline University & 45 & 35.7 \\
\hline \multicolumn{3}{|l|}{ Comorbidities } \\
\hline Hypertension & 34 & 27.0 \\
\hline NIDDM & 9 & 7.1 \\
\hline Heart disease & 14 & 11.1 \\
\hline Lung disease & 10 & 8.0 \\
\hline Enteric disease & 11 & 8.7 \\
\hline Liver disease & 6 & 4.7 \\
\hline Renal failure & 7 & 5.5 \\
\hline Orthopaedic disease & 25 & 19.8 \\
\hline Others & 10 & 8.0 \\
\hline
\end{tabular}

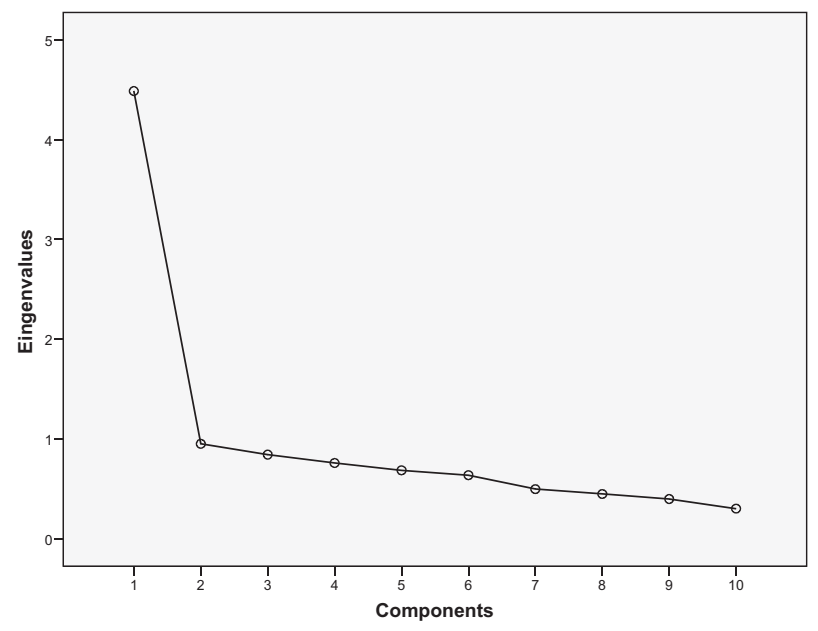

Figure 1. Cattel Scree Test.

\section{Psychometric Scale Properties}

Acceptability. All the questions were well accepted by the patients. The questionnaire was completed in $6.61 \pm$ 2.84 minutes (range: $2-15$ ). Item 8 (sex life) did not receive an answer in $11.9 \%$ of patients; no other missing responses were recorded and no multiple answers were found. There were no problems of comprehension with the questions.

Factor Analysis. Free factor analysis revealed a 1-factor structure on the basis of the numbers of eigenvalues superior to 1, as obtained by Cattel Scree Test (Figure 1). This factor explained $45 \%$ of variance. Item-factor loadings are reported in Table 2.

Reliability. Internal consistency: Cronbach $\alpha$ index for ODI-I was 0.855 . Test-retest stability: correlations between ODI at day 1 and at day 7 demonstrated highly significant (ICC $=0.961$; 95\% CI: 0.943-0.972). Lowest ICC value was achieved for item 3. Repeatability results are fully reported in Table 3.

Validity. Table 4 presents correlations between ODI-I, VAS, RMDQ and SF-36 health survey. (1) Concurrent validity. High correlation was found when ODI-I and VAS were compared $(r=0.730, P<0.001)$. (2) Construct validity. High correlation was found when ODI-I and RMDQ were compared $(r=0.819, P<0.001)$; high

Table 2. Factor Analysis Loadings

\begin{tabular}{lc}
\hline & Components 1 \\
\hline Item 9 & 0.770 \\
Item 10 & 0.738 \\
Item 4 & 0.694 \\
Item 5 & 0.673 \\
Item 6 & 0.664 \\
Item 1 & 0.651 \\
Item 3 & 0.644 \\
Item 8 & 0.635 \\
Item 2 & 0.617 \\
Item 7 & 0.592 \\
\hline
\end{tabular}


Table 3. Repeatability by Intraclass Coefficient Correlation (ICC), 1-7 Day

\begin{tabular}{lcc}
\hline Repeatability & ICC & $95 \% \mathrm{Cl}$ \\
\hline Item 1 & 0.815 & $0.736-0870$ \\
Item 2 & 0.925 & $0.895-0.946$ \\
Item 3 & 0.804 & $0.725-0.861$ \\
Item 4 & 0.944 & $0.920-0.960$ \\
Item 5 & 0.918 & $0.885-0.941$ \\
Item 6 & 0.901 & $0.862-0.929$ \\
Item 7 & 0.831 & $0.768-0.878$ \\
Item 8 & 0.959 & $0.942-0.972$ \\
Item 9 & 0.894 & $0.851-0.924$ \\
Item 10 & 0.930 & $0.902-0.950$ \\
Total & 0.961 & $0.943-0.972$ \\
\hline
\end{tabular}

correlations were moreover found when ODI-I was confronted to each of the 8 SF-36 domains, with the exception of Mental Health $(r=-0.139, P=0.121)$.

\section{Discussion}

We performed and described a study of cross-cultural adaptation, reliability and validity of the Italian version of the Oswestry Disability Index (ODI-I), 2.1a version, in a subacute/chronic LBP population.

Cross-cultural adaptation required a process of translation, backward translation, expert committee revision and test of the prefinal version to warrant that the meaning of the original items were adequately captured in idiomatic translation into the Italian language: steps 1, 2, 3, and 4 indicated that this development was achieved following recommended methodology and guidelines. ${ }^{24,25}$

The questionnaire demonstrated to be highly acceptable, easily understood, and self-administrable, requiring only few minutes to be completed.

The employment of the factor analysis helped to individuate the factorial structure of the Italian version. A 1 -factor solution for ODI-I was identified, explaining $45 \%$ of the variance. It is interesting to note that a factor analysis was performed in 2 other cross-cultural and validation trials, ${ }^{17,19}$ which however, found a 2 -factor structure. Guermazi et al ${ }^{17}$ named their factors as "static physical activities" and "dynamic physical activities" while

\section{Table 4. Concurrent and Construct Validity. Pearson Correlations Between ODI-I, VAS, RMDQ, and SF-36 Domains}

\begin{tabular}{lcc}
\hline Comparison & $r$ & $P$ \\
\hline ODI score vs. VAS & $r=0.730$ & $P<0.001$ \\
ODI score vs. RMDQ & $r=0.819$ & $P<0.001$ \\
ODI score vs. SF-36 domains & $r=-0.751$ & $P<0.001$ \\
SF-36 physical activity & $r=-0.607$ & $P<0.001$ \\
SF-36 physical role & $r=-0.691$ & $P<0.001$ \\
SF-36 physical pain & $r=-0.300$ & $P=0.001$ \\
SF-36 health in general & $r=-0.406$ & $P<0.001$ \\
SF-36 vitality & $r=-0.373$ & $P<0.001$ \\
SF-36 social activities & $r=-0.406$ & $P<0.001$ \\
SF-36 emotional role & $r=-0.139$ & $P=0.121$ \\
SF-36 mental health & & \\
\hline
\end{tabular}

Osthus et al ${ }^{19}$ defined their factors as "pain-related activity" and "pain intensity and pain-related participation."

Internal consistency of ODI-I demonstrated strong correlations $(\alpha=0.855)$. Our finding was higher than Fisher and Johnson $(0.76)^{31}$ and similar to Kopec et al $(0.87)^{32}$ reports in which the original English 2.0 version was implemented. Our results were in agreement to most of the other cross-cultural and adapted versions, ranging from 0.75 (Iranian version) ${ }^{21}$ to 0.94 (Norwegian version). ${ }^{13}$

Test-retest reliability of ODI-I assessed at day 1 and at day 7 demonstrated an highly significant correlation (ICC $=0.961)$; moreover, each item of ODI-I reported satisfying ICC correlations, ranging from 0.804 to 0.959. Our findings were higher than Gronbland et al $(0.83)^{33}$ and Turkish version $(0.938),{ }^{15}$ retested after 1 week; higher results were also found when compared to Danish version (0.91), ${ }^{20}$ retested after 9.1 to 12 days, while similar results were obtained when compared to Swiss-German version (0.96), ${ }^{18}$ retested after 6 days. Due to the natural symptom fluctuation associated with the memory effects, weaker correlations should be considered when comparing our results to Fairbank (0.99, 1 -day retest $),{ }^{8}$ Kopec et al $(0.91,4 \text {-day retest })^{32}$ as well as to other non-English ODI versions, such as Norwegian $(0.88,2$-day retest $),{ }^{13}$ Japanese $(0.93,1$-day retest $),{ }^{14}$ Korean (0.916, 2-day retest), ${ }^{16}$ Arabic (0.98, 3-day retest), ${ }^{17}$ Iranian (0.91, 1-day retest), ${ }^{21}$ and Brazilian $(0.99,1$-day retest $){ }^{22}$

In the determination of concurrent validity, we demonstrated a significant correlation between ODI-I and VAS $(r=0.730, P<0.001)$. Also Gronbland demonstrated a positive correlation when ODI was compared with a VAS $\left(r=0.62\right.$, chronic LBP). ${ }^{33}$ Among translated versions, moderate to good correlations were achieved in Greek $\left(\rho=0.865\right.$, acute/chronic LBP) ${ }^{12}$ Norwegian $(r=$ 0.52 , chronic LBP) ${ }^{13}$ Turkish $\left(r=0.367\right.$, chronic LBP), ${ }^{15}$ Korean $\left(s=0.425\right.$, chronic LBP) ${ }^{16}$ Swiss-German $(r=0.78$, chronic LBP), ${ }^{18}$ Iranian $\left(r=0.54\right.$, chronic LBP), ${ }^{21}$ and Brazilian $(r=0.66 \text {, mainly chronic LBP })^{22}$ populations.

In the analysis for construct validity, we compared ODI-I to RMDQ and to SF-36, respectively. Several studies compared the Oswestry Disability Index with the Roland Morris Disability Questionnaire, showing that the measures could be related; Boscainos et $\mathrm{al}^{34}$ and Leclaire et $a l^{35}$ found a correlation of $r=0.73$ and $r=0.72$, respectively. Our results supported this evidence, as the ODI-I significantly related to the Italian version of the RMDQ $(r=0.819, P<0.001)$. Remarkably, our value was higher than those presented in the other translated forms (Turkish $^{15}: r=0.815$ Brazilian $^{22}: r=0.81$ Danish $^{20}: r=0.78$; Swiss-German ${ }^{18}: r=0.80 ;$ Japanese $^{14}: r=0.785$ Greek $^{12}$ : $\rho=0.729$; Iranian: $r=0.71^{21}$ ).

The ODI was also expected to reveal correlations with health-related quality of life scales. Satisfying results were achieved when ODI-I was compared with each of the 8 domains of SF-36 questionnaire, highly significant with the exception of Mental Health $(r=-0.139, P=$ 0.121). These findings were in agreement with other ex- 
isting studies ${ }^{36-38}$ in which good correlations between ODI and SF-36 were stated. Our findings were also supported by the existing non-English ODI versions, achieving highest correlations when reporting physical function domains and moderate to low correlations when psychosocial scales were analyzed. The Norwegian version $^{13}$ showed better score in Physical Functioning $(r=$ $-0.77)$ and lowest scores in Mental Health $(r=-0.37)$, Emotional Role $(r=-0.33)$, and Vitality $(r=-0.28)$. The Japanese version ${ }^{14}$ found higher values for Physical Role $(r=-0.721)$ and lower scores for Mental Health $(r=-0.603)$. The German version ${ }^{19}$ reported better scores for Physical Function $(r=-0.78)$, lowering when Mental Health and Emotional Role were considered $(r=$ -0.52 and $r=-48$, respectively). The Danish version stated a significant correlation when ODI was compared to Physical Function $(r=-0.75)$ and to Bodily Pain $(r=$ $-0.65) .{ }^{20}$ The Iranian version ${ }^{21}$ demonstrated highest scores in Physical function $(r=-0.68)$ and lowest scores in Emotional Role $(r=-0.38)$, Mental Health $(r=$ -0.36), and General Health $(r=-0.23)$. The Brazilian version $^{22}$ found highest coefficients when Physical Function $(r=-0.83)$ and Bodily Pain $(r=-0.58)$ were considered and lowest scores with Vitality $(r=-0.19)$ and Mental Health $(r=-0.22)$.

\section{- Conclusion}

This study described the development of translation, cultural adaptation, reliability and validity of the Italian version of ODI $2.1 \mathrm{a}$, which is expected to facilitate common low back pain examination and related disability.

This form is recommended to be used for research purposes, with special care to subacute and chronic adult patients, both males and females. Although not examined in the present study, the authors do not find limitations for the implementation of ODI-I in acute subjects and in other causes of low back pain.

Further studies using ODI-I are suggested to focus on minimum detectable change, minimal clinical important difference, sensitivity with specific spinal conditions and discrimination among levels of severity, as already reported for the original version.,

\section{- Key Points}

- The validation of the Italian version of ODI showed good psychometric properties, in particular test-rest stability, internal consistency and validity.

- The factor analysis suggested a 1-factor structure.

- The ODI-I showed good correlation with the VAS as well as the Italian RMDQ and SF-36.

- The ODI-I is recommended to be introduced in the field of low back pain research in Italy.

Supplemental digital content is available for this article. Direct URL citations appear in the printed text, and links to the digital files are provided in the HTML text of this article on the journal's Web site (www.spinejournal.com).

\section{Acknowledgments}

The authors thank Barbara Rocca and Sergio Parazza for the assistance provided with the trial.

\section{References}

1. Koes B, Van Tulder M. Low back pain (acute). Clin Evid 2006;15:1-3.

2. Van Tulder M, Becker A, Bekkering T, et al. European guidelines for the management of acute nonspecific low back pain in primary care. Eur Spine J 2006;15(suppl 2):S169-91.

3. Deyo RA, Weinstein JN. Low back pain. N Engl J Med 2001;344:363-70.

4. Hoogendoorn WE, van Poppel MN, Bongers PM, et al. Systematic review of psychosocial factors at work and private life as risk factors for back pain. Spine 2000;25:2114-25.

5. Bombardier C. Outcome assessments in the evaluation of treatment of spinal disorders: summary and general recommendations. Spine 2000;25:3100-3.

6. Grotle M, Brox JI, Vollestad NK. Functional status and disability questionnaires: what do they assess? A systematic review of back-specific outcome questionnaires. Spine 2005;30:130-40.

7. Roland M, Fairbank J. The Roland-Morris disability questionnaire and the Oswestry disability questionnaire. Spine 2000;25:3115-24.

8. Fairbank J, Couper J, Davies J, et al. The Oswestry low back pain questionnaire. Physiotherapy 1980;66:271-3.

9. Fairbank JCT, Pynsent PB. The Oswestry disability index. Spine 2000;25: 2940-53.

10. Baker DJ, Pynsent PB, Fairbank J. The Oswestry disability index revisited: its reliability, repeatability, and validity, and a comparison with the St. Thomas disability index. In: Roland M, Jenner JR, eds. Back Pain: New Approaches to Rehabilitation and Education. Manchester, United Kingdom: Manchester University Press; 1989:174-86.

11. Oswestry Disability Index homepage. Available at: http://www. orthosurg.org.uk/odi/.

12. Boscainos PJ, Sapkas G, Stillanessi E, et al. Greek versions of the Oswestry and Roland-Morris disability questionnaires. Clin Orthop 2003;411:40-53.

13. Grotle M, Brox JI, Vollestad NK. Cross-cultural adaptation of the Norwegian versions of the Roland-Morris disability questionnaire and the Oswestry disability index. J Rehabil Med 2003;35:241-7.

14. Fujiwara A, Kobayashi N, Saiki K. Association of the Japanese Orthopaedic Association Score with the Oswestry Disability Index, Roland-Morris Disability Questionnaire, and Short-Form 36. Spine 2003;28:1601-7.

15. Yakut E, Duger T, Oksuz C, et al. Validation of the Turkish version of the Oswestry disability index for patients with low back pain. Spine 2004;29: 581-5.

16. Kim DY, Lee SH, Lee HY, et al. Validation of the Korean version of the Oswestry disability index. Spine 2005;30:E123-7.

17. Guermazi M, Mezghani M, Ghroubi S. The Oswestry index for low back pain translated into Arabic and validated in an Arab population. Ann Réadapt Méd Phys 2005;48:1-10.

18. Mannion AF, Junge A, Fairbank JC, et al. Development of a German version of the Oswestry Disability Index, part 1: cross-cultural adaptation, reliability, and validity. Eur Spine J 2005;15:55-65.

19. Osthus H, Cziske R, Jacobi E. Cross-Cultural Adaptation of a German version of the Oswestry disability index and evaluation of its measurement properties. Spine 2006;31:E448-53.

20. Lauridsen HH, Hartvigsen J, Manniche C, et al. Danish version of the Oswestry disability index for patients with low back pain, part 1: cross-cultural adaptation, reliability and validity in two different populations. Eur Spine J 2006 15:1705-16.

21. Mousavi SJ, Parnianpour M, Mehdian H, et al. The Oswestry disability index, the Roland-Morris disability questionnaire, and the Quebec Back Pain Disability Scale: translation and validation studies of the Iranian versions. Spine 2006;31:E454-9.

22. Vigatto R, Alexandre NM, Correa Filho HR. Development of a Brazilian Portuguese Version of the Oswestry Disability Index. Spine 2007;32:481-6.

23. Padua R, Padua L, Ceccarelli E, et al. Cross-cultural adaptation of the lumbar North American Spine Society questionnaire for Italian-speaking patients with lumbar spinal disease. Spine 2001;26:E344-7.

24. Beaton D, Bombardier C, Guilleman F, et al. Recommendations for the Cross-Cultural Adaptation of Health Status Measures. Rosemont, IL; American Academy of Orthopaedic Surgeons, Institute for Work and Health; 1998.

25. Beaton DE, Bombardier C, Guillemin F, et al. Guidelines for the process of cross-cultural adaptation of self-report measures. Spine 2000;25:3186-91.

26. Huskinson EC. Measurement of pain. Lancet 1974;2:1127-31. 
27. Roland M, Morris R. A study of the natural history of back pain: part I. development of a reliable and sensitive measure of disability in low-back pain. Spine 1983;8:141-4.

28. Padua R, Padua L, Ceccarelli E, et al. Italian version of the Roland Disability Questionnaire, specific for low back pain: cross-cultural adaptation and validation. Eur Spine J 2002;11:126-9.

29. Ware JE, Sherbourne CD. The MOS 36-item Short-Form Health Survey (SF-36). Med Care 1992;30:437-83.

30. Apolone G, Mosconi P. The Italian SF-36 Survey: translation, validation and norming. J Clin Epidemiol 1998;51:1025-36.

31. Fisher K, Johnson M. Validation of the Oswestry low back pain disability questionnaire, its sensitivity as a measure of change following treatment and its relationship with other aspects of the chronic pain experience. Physiother Theory Pract 1997;13:67-80.

32. Kopec JA, Esdaile JM, Abrahamowicz M, et al. The Quebec Back Pain Disability Scale: conceptualization and development. J Clin Epidemiol 1996; 49:151-61.

33. Gronblad M, Hupli M, Wennerstrand P, et al. Intercorrelation and test-retest reliability of the Pain Disability Index (PDI) and the Oswestry Disability Questionnaire (ODQ) and their correlation with pain intensity in low back pain patients. Clin J Pain 1993;9:189-95.

34. Boscainos P, Sapkas G, Stilianesi E, et al. Clinical relevance of specific parameters isolated within the Oswestry and Roland-Morris functional disability scales. J Bone Joint Surg (Br) 1999;81-B:239.

35. Leclaire R, Blier F, Fortin L, et al. A cross-sectional study comparing the Oswestry and Roland-Morris functional disability scales in two populations of patients with low back pain of different levels of severity. Spine 1997;22: $68-71$.

36. Grevitt M, Khazim R, Webb J, et al. The short form-36 health survey questionnaire in spine surgery. J Bone Joint Surg (Br) 1997;79:48-52.

37. Taylor S, Taylor A, Foy M, et al. Responsiveness of common outcome measures for patients with low back pain. Spine 1999;24:1805-12.

38. Wittink H, Turk DC, Carr DB, et al. Comparison of the redundancy, reliability, and responsiveness to change among SF-36, Oswestry Disability Index, and multidimensional pain inventory. Clin J Pain 2004;20: $133-42$. 